\title{
Magnetic Fluid Devices for Driving Micro Machines*
}

\author{
Seiichi SUDO**, Yuji TAKAKI***, \\ Yasunari HASHIGUCHI ${ }^{* * *}$ and Hideya NISHIYAMA****
}

\begin{abstract}
This paper is concerned with the development of micro magnetic fluid devices for driving micro machines. Two kinds of new magnetic fluid devices are proposed. One is the magnetic fluid motor, and another one is reciprocating actuator. These micro devices are composed of a permanent $\mathrm{NdFeB}$ magnet and kerosene-based magnetic fluid HC-50. They are characterized by wireless operation with alternating magnetic field. The driving characteristics of micro magnetic fluid devices are examined by using a digital high speed video camera system. The rotary motion of the micro magnetic fluid motor shows the existence of rotational regions and irrotational regions in frequencies of external magnetic field. The amplitude of reciprocating actuator depends on the frequency of magnetic field. The effect of the volume of magnetic fluid adsorbed to the permanent magnet is also revealed experimentally.
\end{abstract}

Key Words: Magnetic Fluid, Magnetic Fluid Motor, Magnetic Fluid Actuator, Micro Device, Micro Actuator, Micro Machine

\section{Introduction}

The last several decades have witnessed an increasing interest in the science and engineering at micro and nanometer length scales ${ }^{(1),(2)}$. With the critical development in micro processing technology and the availability of various functional materials and intelligent material systems, the importance of the developments of micro mechanisms and micro machines is well recognized. Therefore, extensive investigations of this subject have been conducted and reported. For example, the International Symposium on Micro Mechanical Engineering - Heat Transfer, Fluid Dynamics, Reliability and Mechatronics — was held in Tsuchiura and Tsukuba, Japan, by $\operatorname{JSME}^{(3)}$. The main objective of the symposium was to develop micromachines working under highly limited conditions, micro phenomena determing machine performance, and research on utilizing new functions that work on a micro or nano scales. Authors also proposed the micro magnetic swimming mechanism propelled by the alternating

* Received 22nd December, 2004 (No. 04-4267)

** Department of Machine Intelligence and Systems Engineering, Akita Prefectural University, 84-4 Ebinokuchi, Yurihonjo 015-0055, Japan. E-mail: sudo@akita-pu.ac.jp

*** Department of Mechanical Engineering, Iwaki Meisei University, 5-5-1 Iino, Chuohdai, Iwaki 970-8551, Japan

**** Institute of Fluid Science, Tohoku University, 2-1-1 Katahira, Aoba, Sendai 980-8577, Japan.

E-mail: nishiyama@ifs.tohoku.ac.jp magnetic field in a water pipe ${ }^{(4)}$. On the other hand, various devices using magnetic fluids have been developed for the last several decades such as accelerometers, dampers, bearing, pressure seals, loudspeaker coolants et cetera ${ }^{(5)}$. Authors also proposed the novel technique which generates micro droplets and capillary jets of magnetic fluid under magnetic and vibrating fields ${ }^{(6)}$. In spite of many investigations, there is no study on the magnetic fluid micro actuators driven by the alternating magnetic field.

In the present paper, we propose novel micro electromechanical actuator using permanent magnet and magnetic fluid. The micro actuators are composed of small cylindrical $\mathrm{NdFeB}$ magnet and kerosene-based magnetic fluid. Frequency characteristics of micro magnetic fluid actuators are examined with the digital high speed video camera system.

\section{Driving Principle of Magnetic Fluid Devices}

2.1 Element composition of magnetic fluid devices In this paper, we propose two kinds of new magnetic fluid devices. One is the micro magnetic fluid reciprocator, and another is the micro magnetic fluid motor. These devices are composed of $\mathrm{NdFeB}$ magnet and kerosene-based magnetic fluid (ferricolloid HC-50). Table 1 shows the physical properties of elements of magnetic fluid reciprocator. Table 2 shows the physical properties of elements of magnetic fluid motor. In this experiment, $\mathrm{Nd}_{2} \mathrm{Fe}_{14} \mathrm{~B}$ permanent magnets with excellent magnetic characteristics are used. Table 3 shows the magnetic properties of 
Table 1 Physical properties of magnetic fluid reciprocator

\begin{tabular}{|c|c|}
\hline Permanent magnet & NdFeB magnet \\
\hline Diameter of magnet & $4 \mathrm{~mm}$ \\
\hline Length of magnet & $2 \mathrm{~mm}$ \\
\hline $\begin{array}{c}\text { Magnetic flux density of } \\
\text { magnet }\end{array}$ & $330 \mathrm{mT}$ \\
\hline $\begin{array}{c}\text { Magnetic fluid } \\
\text { Saturation magnetization of } \\
\text { magnetic fluid }\end{array}$ & Ferricolloid HC-50 \\
\hline $\begin{array}{c}\text { Viscosity of magnetic fluid } \\
\text { furface tension of magnetic } \\
\text { fluid }\end{array}$ & $93 \mathrm{mPa} \cdot \mathrm{s}$ \\
\hline
\end{tabular}

Table 2 Physical properties of magnetic fluid motor

\begin{tabular}{|c|c|}
\hline Permanent magnet & NdFeB magnet \\
\hline Diameter of magnet & $5.5 \mathrm{~mm}$ \\
\hline Length of magnet & $2.5 \mathrm{~mm}$ \\
\hline $\begin{array}{c}\text { Magnetic flux density of } \\
\text { magnet }\end{array}$ & $390 \mathrm{mT}$ \\
\hline Magnetic fluid & Ferricolloid HC-50 \\
\hline $\begin{array}{c}\text { Saturation magnetization of } \\
\text { magnetic fluid }\end{array}$ & $93.42 \mathrm{kA} / \mathrm{m}$ \\
\hline Viscosity of magnetic fluid \\
\hline $\begin{array}{c}\text { Surface tension of magnetic } \\
\text { fluid }\end{array}$ & $0.0277 \mathrm{~N} / \mathrm{m}\left(\right.$ at $\left.19^{\circ} \mathrm{C}\right)$ \\
\hline
\end{tabular}

Table 3 Magnetic properties of $\mathrm{NdFeB}$ magnet

\begin{tabular}{|c|c|c|c|c|}
\hline \multirow{2}{*}{$\begin{array}{c}\text { Magnet } \\
\text { material }\end{array}$} & \multirow{2}{*}{$\begin{array}{c}\text { Residual magnetic } \\
\text { flux density } \mathrm{Br}(\mathrm{T})\end{array}$} & \multicolumn{2}{|c|}{ Coercive force } & Maximum energy \\
\cline { 3 - 4 } & $\mathrm{bHC}(\mathrm{kA} / \mathrm{m})$ & $\mathrm{iHC}(\mathrm{kA} / \mathrm{m})$ & $\begin{array}{c}\text { product } \\
(\mathrm{BH})_{\max }\left(\mathrm{kJ} / \mathrm{m}^{3}\right)\end{array}$ \\
\hline $\mathrm{Nd}_{2} \mathrm{Fe}_{14} \mathrm{~B}$ & $1.62-1.33$ & $859-970$ & $>955$ & $302-334$ \\
\hline
\end{tabular}

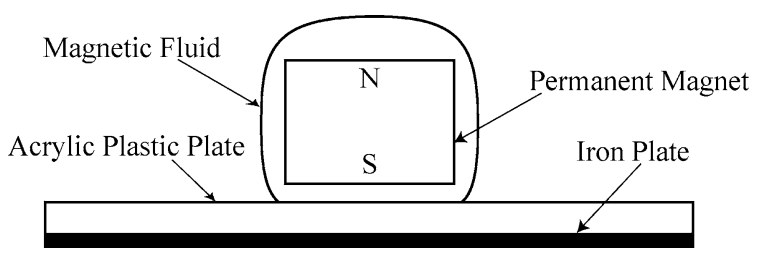

Fig. 1 Basic structure of magnetic fluid device

the magnet material. The fundamental structure of magnetic fluid devices is shown in Fig. 1. Magnetic fluid is adsorbed to the permanent magnet. The magnet-magnetic fluid element is put on the acrylic plastic base with $5 \mathrm{~mm}$ thickness. The iron plate with $1 \mathrm{~mm}$ thickness is laid under the acrylic plastic base for the weak attraction of the
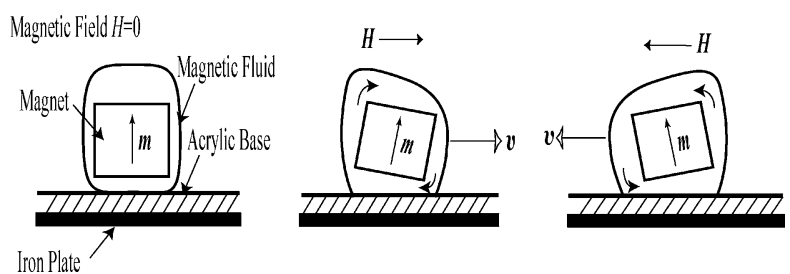

(a) Driving principle of magnetic fluid reciprocator
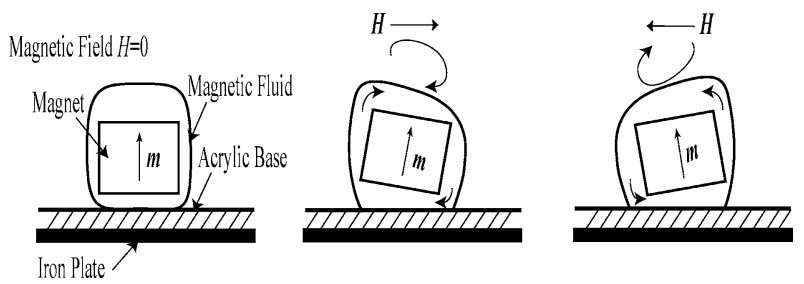

(b) Driving principle of magnetic fluid motor

Fig. 2 Schematic picture of driving principle

magnet-magnetic fluid element. The weak magnetic attraction keeps the magnet-magnetic fluid element at a constant state against the base incline. The iron plate plays an important role as the stabilizer for the actuators. The viscosity of magnetic fluid relates to friction of magnetmagnetic fluid element and acrylic plastic base. However, the viscous effect in magnetic fluid devices can be neglected, because the viscosity of kerosene-based magnetic fluid is very low.

\subsection{Driving principle of magnetic fluid device}

As shown in Fig. 1, a phenomenon of self-levitation occurs when a permanent magnet is immersed in magnetic fluid. The field surrounding the magnet is symmetrically disposed, so the pressure in the magnetic fluid is symmetrically distributed, and the magnet experiences an equilibrium of magnetic forces when the magnet is centered ${ }^{(5)}$. Self-levitation of a permanent magnet in magnetic fluid is attributed to the magnetic force ${ }^{(5)}$ :

$$
\boldsymbol{F}_{m}=-V \mu_{0} M \nabla H_{\mathrm{m}}
$$

where $V$ is the volume of magnet, $\mu_{0}$ is the permeability of free space, $M$ is the intensity of magnetization, and $H_{\mathrm{m}}$ is the magnetic field. Self-levitating magnet in magnetic fluid can move by the external force very easily, because the magnetic fluid plays the role of lubrication. The driving principle of magnetic fluid device in the external alternating magnetic field is shown in Fig. 2. The magnetic torque $\boldsymbol{T}$ acting on the permanent magnet with magnetic moment $\boldsymbol{m}$ in the external magnetic field $\boldsymbol{H}_{e}$ is given by Eq. (2):

$$
\boldsymbol{T}=\boldsymbol{m} \times \boldsymbol{H}_{e}
$$

The permanent magnet in magnetic fluid shows the rotational oscillation, according to the direction of the alternating magnetic field as shown in Fig. 2. In this experiment, the external magnetic field is given in the sinusoidal form:

$$
\boldsymbol{H}_{e}=H_{0} \boldsymbol{e} \sin \left(2 \pi f_{0} t\right)
$$


where $H_{0}$ is the amplitude of alternating magnetic field, $\boldsymbol{e}$ is a unit vector, $f_{0}$ is the excitation frequency, and $t$ is the time. The magnet-magnetic fluid element is moved in the unidirection magnetic field of long duration at lower excitation frequencies (Fig. 2 (a)). The rotational motion produced by Eq. (2) brings about nonuniform magnetic field. Therefore, the translation force $\boldsymbol{F}_{t}$ acts upon the permanent magnet:

$$
\boldsymbol{F}_{t}=m_{x} \frac{\partial \boldsymbol{H}_{i}}{\partial x}+m_{y} \frac{\partial \boldsymbol{H}_{i}}{\partial y}+m_{z} \frac{\partial \boldsymbol{H}_{i}}{\partial z}
$$

where $(x, y, z)$ is an orthogonal coordinate system, $\boldsymbol{H}_{i}$ is the magnetic field, and suffix $x, y, z$ shows each component. The translation force $\boldsymbol{F}_{t}$ causes the reciprocating motion of the magnet-magnetic fluid element.

On the other hand, reciprocating motion is not generated at higher frequencies. The magnetic field of short duration at higher frequencies is not sufficient in order to move the magnet-magnetic fluid element. In other words, the moving distance of the element is very short, because the action time of the magnetic field in one direction is $1 / f_{0}$. Therefore, the permanent magnet in magnetic fluid swings with great regularity at higher excitation frequencies. However, the permanent magnet rotates under some conditions. The permanent magnet rotates, when the energy of the rotary motion is lower compared to the energy of swing motion (Fig. 2(b)).

\section{Experimental Apparatus and Procedures}

The experimental apparatus is composed of magnetic fluid device, magnetic field generation system, and highspeed video camera system. A schematic block diagram of the experimental apparatus to study frequency characteristics of magnetic fluid devices driven by the alternating magnetic field is shown in Fig. 3. The magnetic fluid devices are composed of a cylindrical $\mathrm{NdFeB}$ permanent magnet and magnetic fluid. The magnetic fluid motor has four vanes for the application and visualization of the rotational motion. The alternating magnetic field is generated by applying alternating current voltage to the Helmholtz coil. The alternating current signal is supplied from the frequency synthesizer. Figure 4 shows the photograph of the magnetic fluid device and the magnetic field generation system. The magnetic fluid device is located on a center part of the Helmholtz coil. The direction of the magnetic field is parallel to the horizontal acrylic plastic base through all the experiments. Figure 5 shows the rough sketch of the magnet with four vanes. The rotational axis of the magnetic fluid motor is shown in Fig. 5 (a), and the direction of the rotation is shown in overhead view Fig. 5 (b). In the experiment, a constant volume of magnetic fluid was adsorbed by the magnet. Frequency characteristics of magnetic fluid devices are studied with the high-speed video camera system. The video camera system is composed of a high-speed video camera, a video

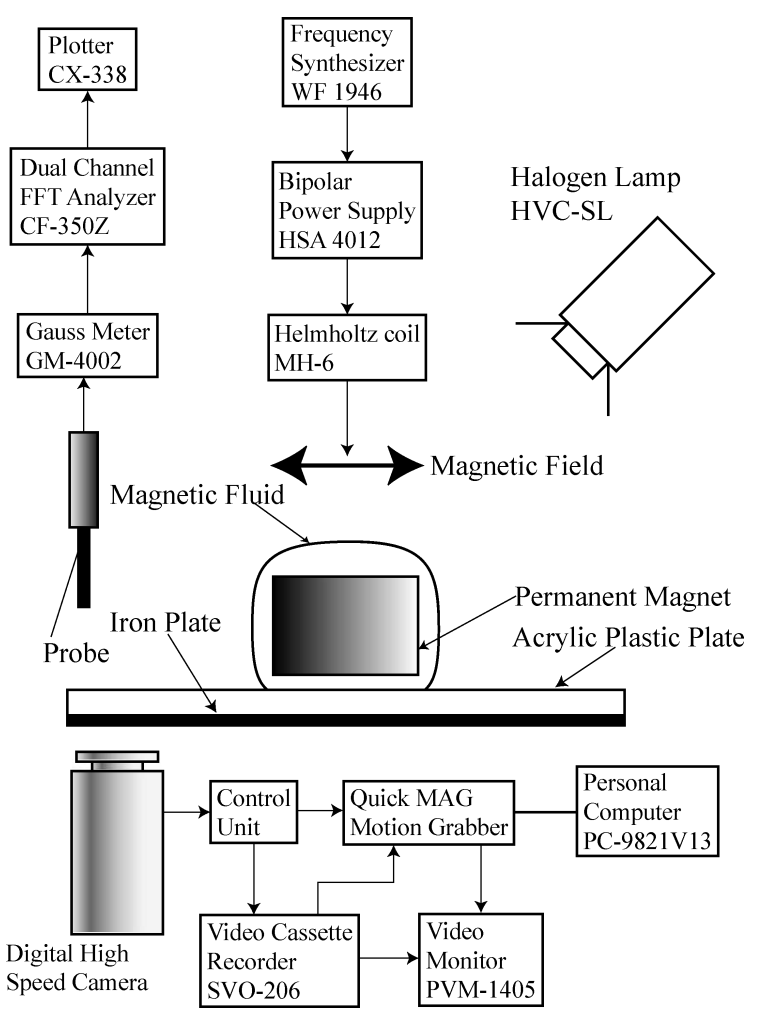

Fig. 3 A schematic diagram of experimental apparatus

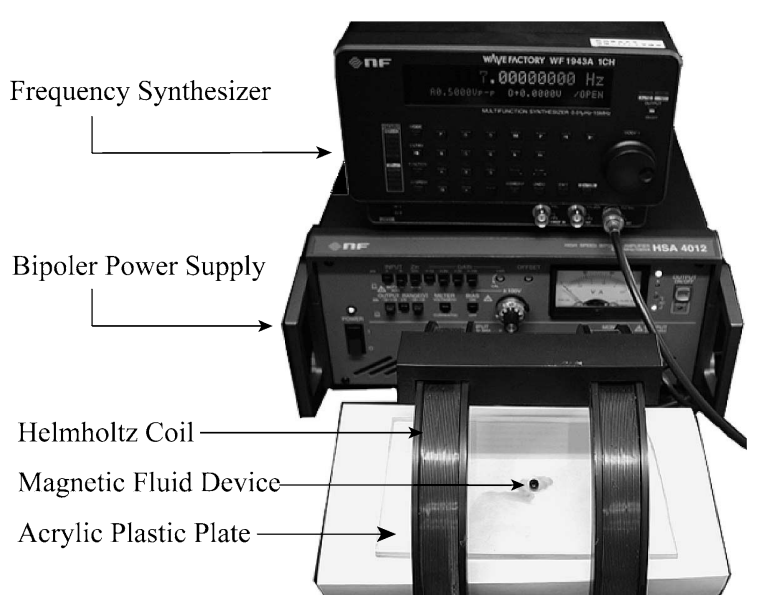

Fig. 4 The photograph of the magnetic fluid device and the magnetic field generation system

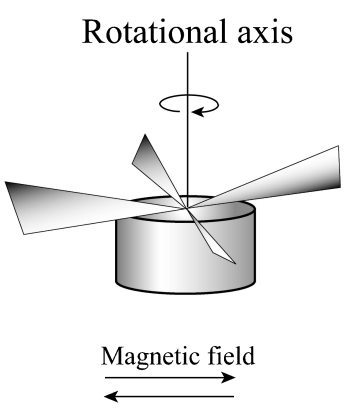

(a)

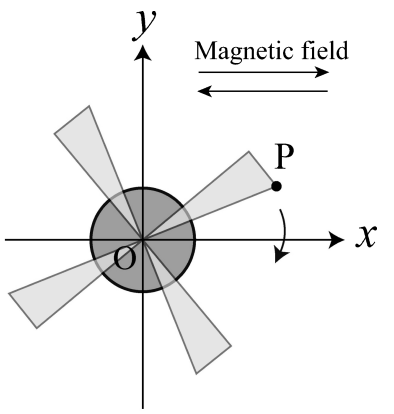

(b)
Fig. $5 \mathrm{NdFeB}$ magnet with four vanes for magnetic fluid motor 
cassette recorder, a motion grabber, a video monitor, and a personal computer ${ }^{(6)}$.

In this experiment, the applied voltage is given in the sinusoidal form as follows,

$$
E_{c}=\frac{E}{2} \sin \left(2 \pi f_{0} t\right)
$$

where $E / 2$ is the amplitude of alternating voltage. The magnetic field is generated at a constant voltage $E$ ( $E$ is the total amplitude of alternating voltage). The direction of magnetic field is parallel to the horizontal acrylic plastic base. Frequency characteristics of magnetic fluid devices are obtained for various applied voltages.

\section{Micro Magnetic Fluid Motor}

The micro magnetic fluid motor driven by the alternating field is proposed in this paragraph. The rotary type device is composed of a cylindrical $\mathrm{NdFeB}$ magnet with four vanes and magnetic fluid. The magnetic fluid device shows the rotational motion, when the magnet-magnetic fluid element is subjected to external alternating magnetic field. Figure 6 shows the relationship between input frequency $f_{0}$ and output angular frequency $\omega_{\mathrm{e}}$. The white circle plotting data show the case of the ascending condition in the frequency of alternating magnetic field. The black circle plotting data show the case of the descending condition in the external frequency. In Fig. 6, the total amplitude of input voltage is $E=3 \mathrm{~V}$, and the volume of magnetic fluid adsorbed to the permanent magnet is $V_{\mathrm{m}}=1.0 \times 10^{-7} \mathrm{~m}^{3}$. The arrows show the orbit of the experimental condition in the input frequency. It can be seen from Fig. 6 that a rotation response of micro magnetic fluid motor has hysteresis. Figure 7 shows the effect of applied voltage on the frequency characteristics of magnetic fluid motor. The increase of applied voltage expands the rotational region of magnetic fluid motor. In Fig. 7, $\omega_{0}$ is the input angular rotational frequency $\left(\omega_{0}=2 \pi f_{0}\right)$. Input angular frequencies $\omega_{0}$ and output angular frequencies $\omega_{\mathrm{e}}$ agree precisely in Fig. 7.

Frequency characteristics of micro magnetic fluid

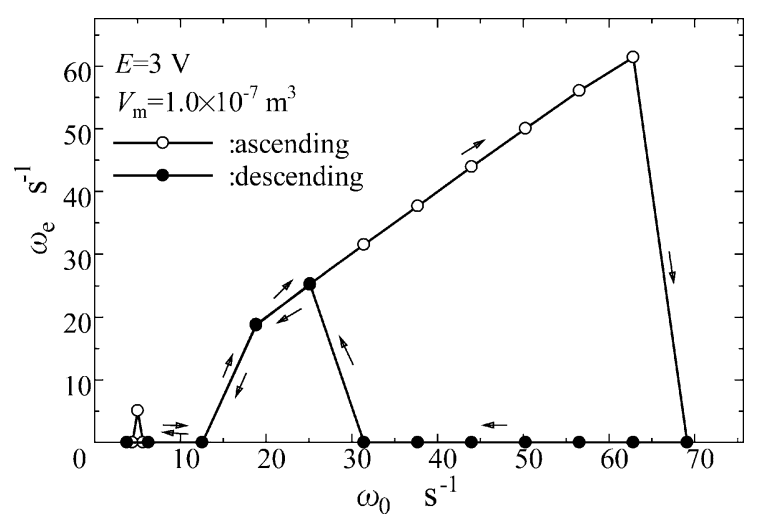

Fig. 6 Relation between rotary angular frequency and field frequency motor were effected by experimental conditions such as volume of magnetic fluid, $V_{\mathrm{m}}$ or applied voltage $E$. Figure 8 shows frequency characteristics of magnetic fluid motor at volume $V_{\mathrm{m}}=1.5 \times 10^{-7} \mathrm{~m}^{3}$. It can be seen form Fig. 8 that there are two rotating regions in frequency characteristic curve of magnetic fluid motor. The higher rotating frequency region expands with the increase of the
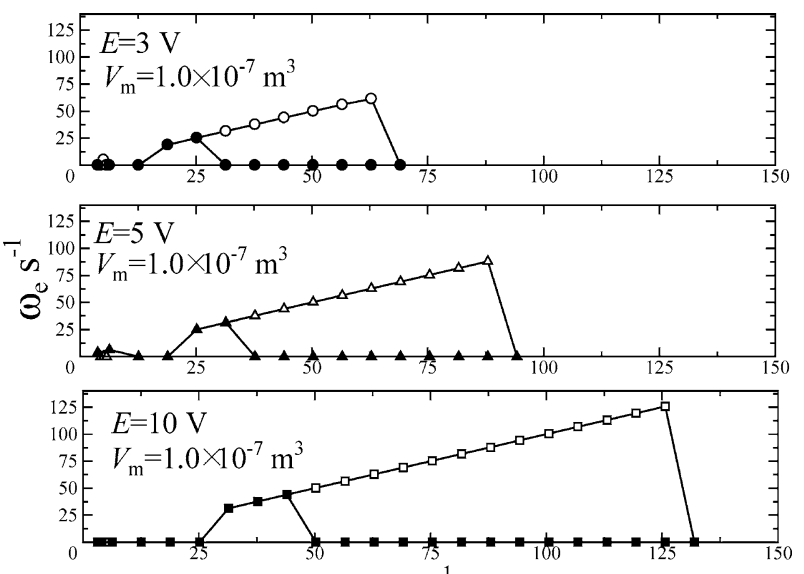

$\omega_{0} \mathrm{~s}^{-1}$

Fig. 7 Effect of applied voltage on frequency characteristics

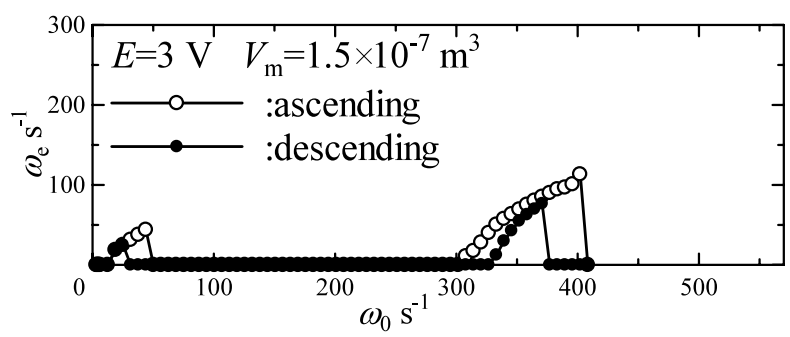

(a) Applied voltage $E=3 \mathrm{~V}$

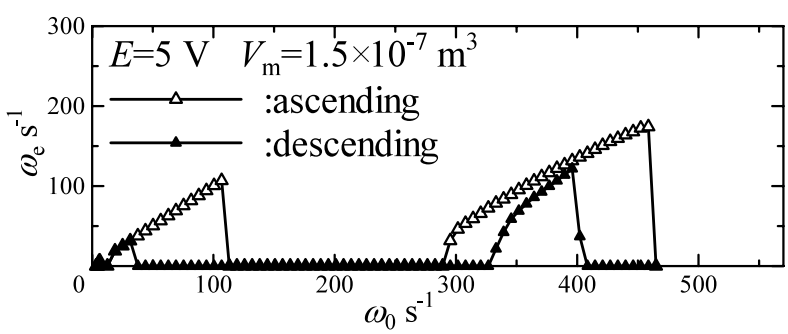

(b) Applied voltage $E=5 \mathrm{~V}$

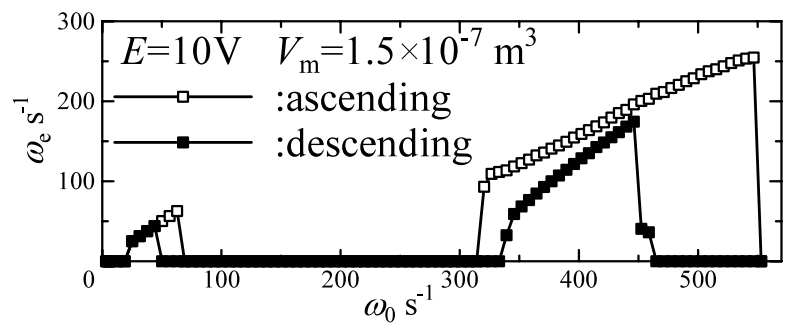

(c) Applied voltage $E=10 \mathrm{~V}$

Fig. 8 Frequency characteristics of magnetic fluid motor $\left(V_{\mathrm{m}}=1.5 \times 10^{-7} \mathrm{~m}^{3}\right)$ 
applied voltage $E$. However, the lower frequency region is reduced at $E=10 \mathrm{~V}$. This fact shows that the optimum voltage exists in the rotary motion of magnetic fluid motor. In the experiment, it was observed that the permanent magnet in magnetic fluid rotated perpendicularly (90 degrees) in the higher applied magnetic fields. In the higher rotating frequency region, the rotational frequency of magnetic fluid motor dose not agree with the frequency of magnetic field. The cause of this disagreement in the frequency is uncertain. In the lower rotating frequency region, however, the rotational frequency of magnetic fluid motor exactly agrees with the frequency of magnetic field.

\section{Micro Magnetic Fluid Reciprocator}

The micro magnetic fluid reciprocator driven by the alternating field is proposed in this paragraph. This is a linearly displacement type actuator composed of a small cylindrical permanent magnet and magnetic fluid as shown in Fig. 1. When the system is subjected to the alternating field with lower frequencies, the magnet-magnetic fluid element shows reciprocating motion for right and left along the direction of magnetic field. The magnetic fluid adsorbed to the permanent magnet plays the role of the lubrication of element sliding. Figure 9 shows the definition of the total amplitude of reciprocating motion, $S$. The relationship between magnetic field and total ampli-

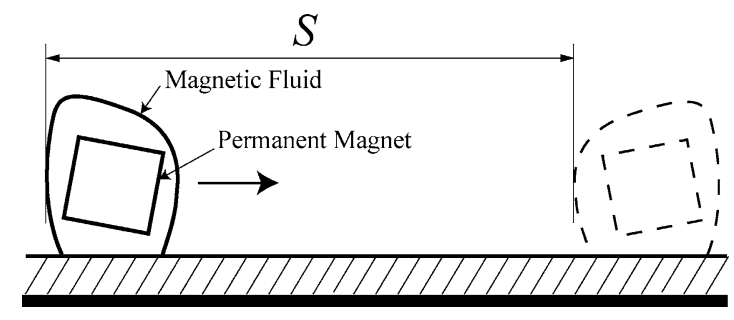

Fig. 9 Reciprocating motion and definition of $S$

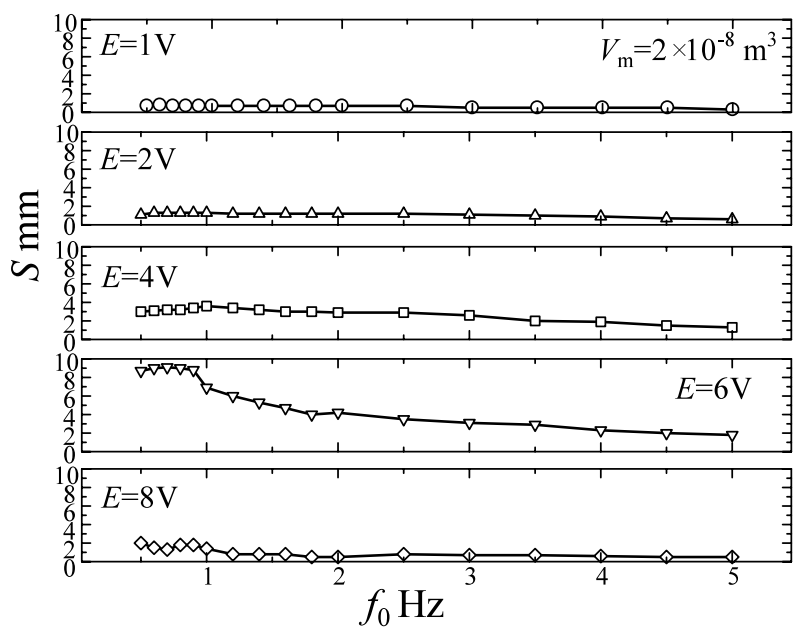

Fig. 10 Frequency characteristics of magnetic fluid reciprocator $\left(V_{\mathrm{m}}=2 \times 10^{-8} \mathrm{~m}^{3}\right)$ tude was tested in the experiment. Figure 10 shows frequency characteristics of micro magnetic fluid reciprocator. In Fig. 10, the volume of the magnetic fluid adsorbed to the magnet is $V_{\mathrm{m}}=2 \times 10^{-8} \mathrm{~m}^{3}$. It can be seen from Fig. 10 that frequency characteristics of magnetic fluid reciprocator depend on applied voltage $E$. The total amplitude $S$ is increased with the increase of $E$ at the constant frequency under the range of voltage $E=6 \mathrm{~V}$. On the other hand, the total amplitude at $E=8 \mathrm{~V}$ is decreased comparing with the condition of $E=6 \mathrm{~V}$. This fact shows the existence of optimum condition in the applied voltage. The response of permanent magnet in magnetic fluid is very different from normal reciprocating motion over $E=6 \mathrm{~V}$. Figure 11 shows also frequency characteristics of micro magnetic fluid reciprocator with $V_{\mathrm{m}}=4 \times 10^{-8} \mathrm{~m}^{3}$. The increase of the volume of magnetic fluid increases the mobility of permanent magnet, because the attractive force between magnet and iron plate decreases. Therefore, frequency characteristic curves shift for higher values comparing with $V_{\mathrm{m}}=2 \times 10^{-8} \mathrm{~m}^{3}$. It can be seen from Fig. 11

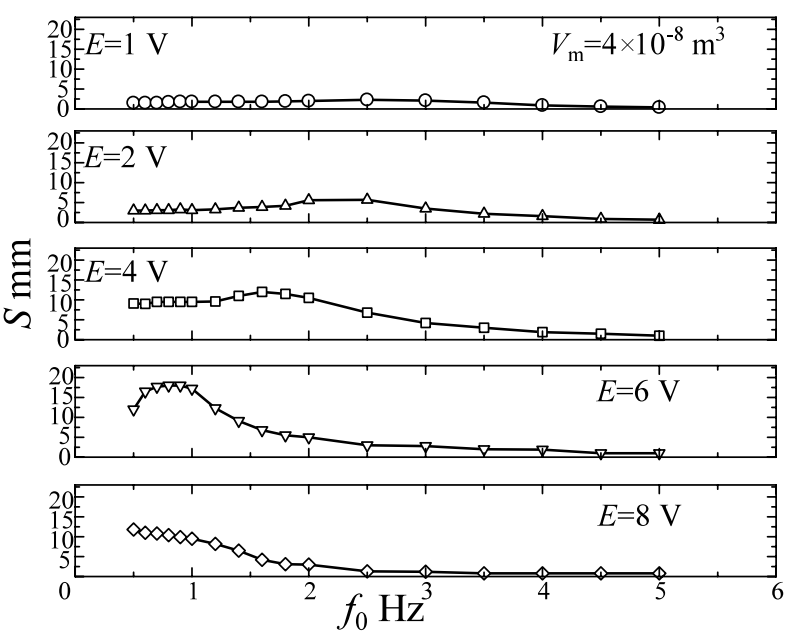

Fig. 11 Frequency characteristics of magnetic fluid reciprocator $\left(V_{\mathrm{m}}=4 \times 10^{-8} \mathrm{~m}^{3}\right)$

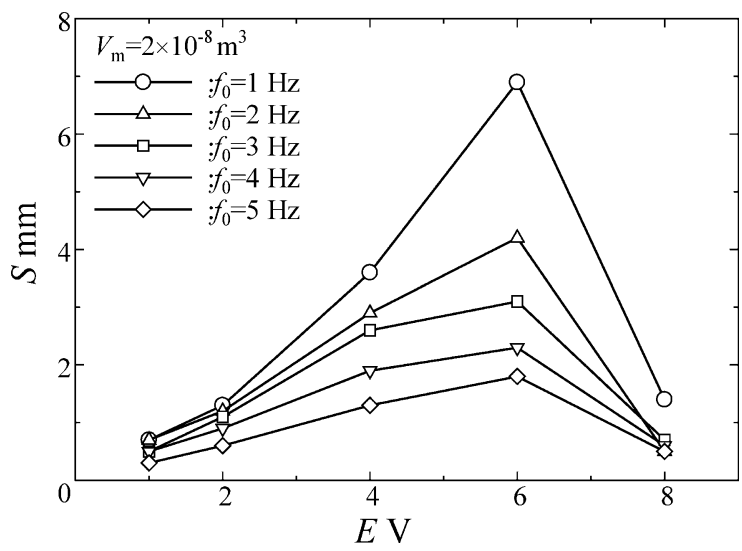

Fig. 12 Effect of applied voltage on reciprocating amplitude $\left(V_{\mathrm{m}}=2 \times 10^{-8} \mathrm{~m}^{3}\right)$ 


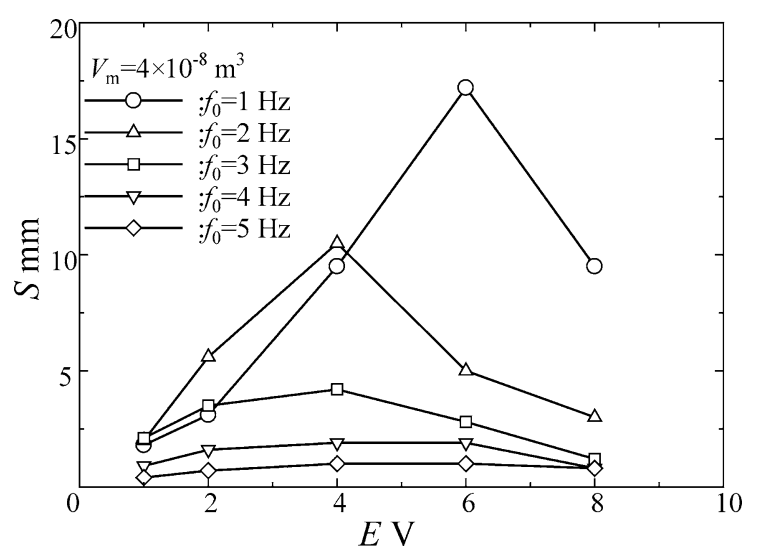

Fig. 13 Effect of applied voltage on reciprocating amplitude $\left(V_{\mathrm{m}}=4 \times 10^{-8} \mathrm{~m}^{3}\right)$

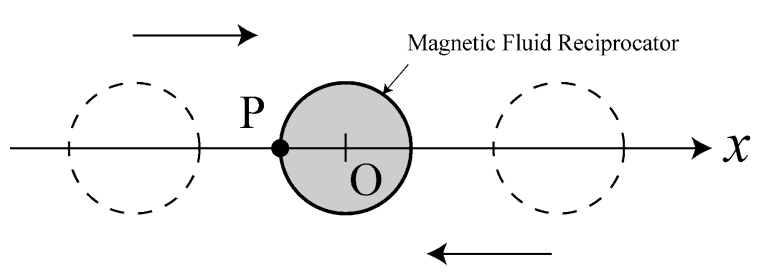

Fig. 14 One dimensional coordinate system and point $\mathrm{P}$

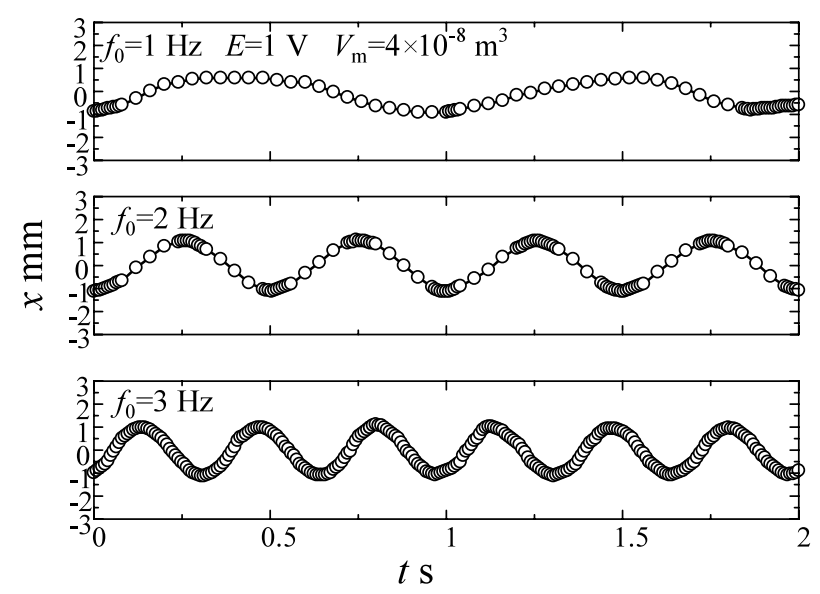

Fig. 15 Reciprocating motion at various frequencies

that frequency characteristics curves have peak values at some specific frequencies of alternating magnetic field. Figure 12 shows the effect of the applied voltage on the total amplitude of reciprocating motion. The parameter in Fig. 12 is the frequency of alternating magnetic field. The curves of each frequency show the maximum values at the applied voltage $E=6 \mathrm{~V}$. Figure 13 shows also the effect of the applied voltage on the total amplitude at the volume $V_{\mathrm{m}}=4 \times 10^{-8} \mathrm{~m}^{3}$. The applied voltage which shows the maximum value is different by the each frequency curve. It seems that the frequency of peak value of frequency characteristic curves is a natural frequency of reciprocating motion of magnetic fluid actuator. Reciprocating trajectory of magnetic fluid device was examined under var-

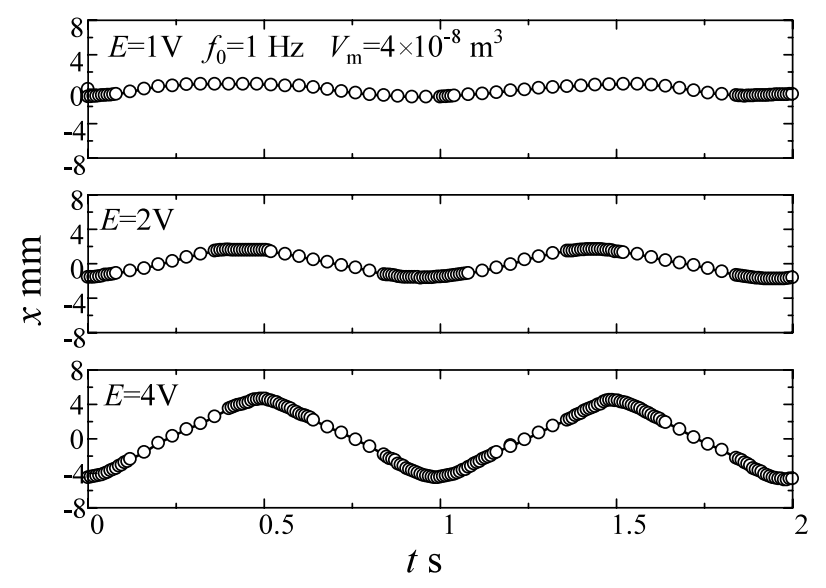

Fig. 16 Reciprocating motion in various voltages

ious excitation conditions. Figure 14 shows one dimensional coordinate system for the motion analysis. The point $\mathrm{P}$ in Fig. 14 is plotted against the time. Figure 15 shows the reciprocating motion at the various frequencies. The frequency of the reciprocating motion exactly agrees with the frequency of the applied magnetic field. In the experimental condition of Fig. 15, the amplitude of reciprocating motion is almost equal regardless of the frequency of the applied field. Figure 16 shows the reciprocating motion in various applied voltages at $f_{0}=1 \mathrm{~Hz}$. It can be seen from Fig. 16 that the amplitude of reciprocating motion increases with the increase of the applied voltage. The increase of the voltage $E$ means the increase of the magnetic field intensity.

\section{Conclusions}

Novel micro device using permanent magnet and magnetic fluid were proposed, and examined from the viewpoint of frequency characteristics. The results obtained are summarized as follows:

(1) The micro magnetic fluid motor shows the stable rotation at the specific frequency range. Frequency characteristics of magnetic fluid motor show the hysteresis in the relation between input and output angular frequencies. Frequency characteristics depend on the applied voltage to the Helmholtz coil and the volume of magnetic fluid adsorbed to the permanent magnet.

(2) The micro magnetic fluid reciprocator shows stable reciprocating motion with large amplitude at lower frequency range. Frequency characteristics of magnetic fluid reciprocator depend on the applied voltage to the Helmholtz coil and the volume of magnetic fluid. There are optimum values for the reciprocating motion with larger amplitude in the applied voltage and the volume of magnetic fluid.

\section{References}

( 1 ) Esashi, M., Micromachines, (in Japanese), (2002), pp.1-669, Sangyo Gijutsu Service Center. 
( 2 ) Higuchi, T., Micromachine Technologies, (in Japanese), (2003), pp.1-753, Sangyo Gijutsu Service Center.

( 3 ) JSME, Proc. Int. Symp. Micro-Mech. Engn., (2003), pp.1-520.

( 4 ) Sudo, S., Orikasa, R. and Honda, T., Locomotive Characteristics of Swimming Mechanism Propelled by Alternating Magnetic Field, Int. J. Appl. Electromagn.
Mech., Vol.19 (2004), pp.263-267.

( 5 ) Rosensweig, R.E., Ferrohydrodynamics, (1985), pp.1344, Cambridge University Press.

( 6 ) Sudo, S. and Ise, K., Droplet Production from a Capillary Jet of Magnetic Fluid under a Magnetic Field, Int. J. Appl. Electromagn. Mech., Vol.19 (2004), pp.169174. 